\title{
Polarimetry of Saturnian satellite Enceladus
}

\author{
S. V. Zaitsev ${ }^{1 *}$, N. N. Kiselev ${ }^{1}$, V. K. Rosenbush ${ }^{1}$, S. V. Kolesnikov², D. N. Shakhovskoy ${ }^{3}$ \\ ${ }^{1}$ Main Astronomical Observatory of the NAS of Ukraine, Akademika Zabolotnoho Str., 27, 03680, Kyiv, Ukraine \\ ${ }^{2}$ Astronomical Observatory of Odesa National University, T. G. Shevchenko Park, 65014, Odesa, Ukraine \\ ${ }^{3}$ Crimean Astrophysical Observatory, 98409, Nauchny, Crimea, Ukraine
}

\begin{abstract}
We present results of polarimetric observations of Saturn's moon Enceladus carried out from April 14, 2010 to April 13, 2013 in WR spectral band $(550-750 \mathrm{~nm})$. We used 2.6-m telescope equipped with a one-channel photoelectric photometer-polarimeter (Crimean Astrophysical Observatory). The measurements were performed at phase angles ranging from $1.65^{\circ}$ to $5.71^{\circ}$. The phase-angle dependence of linear polarization of Enceladus was obtained using the results of our observations. Results obtained are discussed in terms of existing models of light scattering by regolith surfaces.
\end{abstract}

Key words: polarization, planets and satellites: surfaces

\section{INTRODUCTION}

Enceladus is one of the most interesting planetary satellites in the Solar System. Its diameter is $504 \mathrm{~km}$ and its average density is approximately $1.61 \mathrm{~g} / \mathrm{cm}^{3}$ [19]. The geometrical albedos of its leading and trailing hemispheres at $900 \mathrm{~nm}$ are 1 and 1.06 [18], respectively, which are the highest geometrical albedos in the Solar System. Enceladus orbits Saturn at a distance of 3.95 saturnian radii and is embedded in the large diffuse E-ring which has the highest density near Enceladus' orbit. Photometric and spectroscopic observations established that Enceladus' surface is nearly pure water ice [3, 4, 5, 25], but a possible detection of surface $\mathrm{NH}_{3}$ ice and ammonia has also been reported $[9,12,26]$. The satellite exhibits both cryovolcanic and tectonic activity across its geologically young South Polar Terrain [19, 24, 28]. The discovery of jets of water vapour and ice grains emanating from Enceladus' south pole is one of the major highlights of the Cassini-Huygens mission [7, 27].

The photometric phase dependence of Enceladus exhibits a nonlinear enhancement of brightness at low solar phase angles $[18,25]$. This low phase angle phenomenon is known as brightness opposition effect. According to [16], brightness opposition effect for high-albedo atmosphereless Solar System bodies should be accompanied by sharp peak of negative linear polarization of the same angular width centred at a very small phase angle superimposed on the regular negative polarization branch. It is socalled polarization opposition effect (POE) [13, 16]. Its angular width and depth depend on the sizes and albedo of the reflecting surface's particles, their refractive index and packing density. A narrow and sharp peak of negative polarization at nearly zero phase angles was first measured in $[10,15]$ for a particulate surface composed of microscopic $\mathrm{MgO}$ and $\mathrm{Al}_{2} \mathrm{O}_{3}$ grains, during a laboratory study of the dependence of the polarization phase curve on the size of the grains forming a reflecting surface.

Polarization opposition effect in a form of narrow secondary minimum of negative polarization at small phase angles was detected for high-albedo Jovian satellites Io, Europa, Ganymede [20, 21, 30], and for E-type asteroids 44 Nysa [22] and 64 Angelina $[22,29]$. For Saturnian satellites Rhea [31] and bright side of Iapetus [8] POE was detected in a form of asymmetrical negative polarization branch.

Despite the long history of observations and unique characteristics of Enceladus, polarimetrical observations of the satellite have not been carried out. The reason is that Enceladus is a very difficult object to observe due to its close proximity to Saturn's disc. It can only be observed near the greatest eastern and western elongations, when it is approximately 40 arcsec far from Saturn's centre.

Studying the phase-angle dependence of linear polarization of Saturn's high-albedo satellite Enceladus is very important for discerning the physical characteristics of its surface. We present the results of the first polarimetric observations of Enceladus.

\section{OBSERVATIONS}

Polarimetric observations of Enceladus were carried out over a period of seven nights from April 14, 2010 through April 13, 2013. We used 2.6-m Shain telescope equipped with a one-channel photoelectric photometer-polarimeter [29] (Crimean Astrophysical Observatory). The photopolarimeter is based on modulation principle with a rapidly rotating re-

\footnotetext{
* sergio908@rambler.ru

(C) S. V.Zaitsev, N. N. Kiselev, V.K.Rosenbush, S. V. Kolesnikov, D. N. Shakhovskoy, 2015
} 
tarder. It consists of a motionless Glan prism installed immediately behind an achromatic quarterwave phase plate rotating with a frequency of approximately $33 \mathrm{~Hz}$. The principle of synchronous detection is used. The intensity components used to compute the Stokes parameters $Q, U, V$ are selected by eight virtual counters (one physical counter), which are switched on sequentially and accumulate the signal for $8 \times 2$ position angles of each rotation of the phase plate $\left(0^{\circ}-22^{\circ} .5,22^{\circ} .5-45^{\circ}, \ldots, 337^{\circ} .5-\right.$ $\left.360^{\circ}\right)$. The observations were carried out at phase angles ranging from $1.65^{\circ}$ to $5.71^{\circ}$ in the WR spectral band $(550-750 \mathrm{~nm})$. The measurements were performed in the mode of linear polarization.

The sky's background intensity was measured before and after each set of Enceladus measurements. These values were interpolated and reduced from each measurement of the satellite in each of eight virtual counters. In order to take into account the variation of the sky's background intensity and polarization (because of close Saturn's disc and rings), the background intensity was measured in two places equally spaced away from the satellite in the plane perpendicular to the orbital plane. We used 10 and 15 arcsec diaphragms depending on the observational circumstances.

The normalized Stokes parameters of linear polarization for the satellite were computed as:

$$
\begin{aligned}
& u=u_{o}-\bar{u}_{\text {inst }}, \\
& q=q_{o}-\bar{q}_{\text {inst }},
\end{aligned}
$$

where $u_{o}, q_{o}$ are the average values of measured normalized Stokes parameters of linear polarization of Enceladus for each night, and $\bar{u}_{\text {inst }}, \bar{q}_{\text {inst }}$ are the instrumental polarization parameters determined from observations of unpolarized standard stars taken from [11].

The degree of linear polarization $P$ was calculated using the Stokes parameters $u$ and $q$ received from observations:

$$
P=\sqrt{u^{2}+q^{2}}
$$

The position angle of the polarization plane $\theta$ of the satellite was calculated using the expression:

$$
\theta=\frac{1}{2} \arctan \left(\frac{u}{q}\right)+\Delta \theta
$$

where $\Delta \theta$ is the requisite correction for the zero point of the position angle of the polarization plane. It was calculated as the average value of the differences between the measured position angles of the polarization plane $\theta_{o}$ of the standard stars with well-known large interstellar polarization and their position angles $\theta_{\text {cat }}$ taken from the polarimetrical catalogue [11]:

$$
\Delta \theta=\theta_{o}-\theta_{\text {cat }} .
$$

The zero-point of the position angle of the polarization plane was stable within $2^{\circ}$.
The random errors of $u$ and $q$ were calculated in two ways. One way was by the statistics of recorded photons from the object according to the expression:

$$
\sigma_{u}^{s t}=\sigma_{q}^{s t}=\frac{\pi 100}{2} \sqrt{\frac{1}{N\left(1+\frac{1+\tau}{R}\right)}},
$$

where $N=\sum_{i=1}^{8} N_{i}$ is the sum of the object photons recorded in eight counters, $\tau$ is a ratio of times of measurements of object and the sky background, and $R$ is a ratio of the recorded photons from the object and from the sky background for the same time [23]. The other way was using the mean square errors $\sigma_{u}^{d}$ and $\sigma_{q}^{d}$ of the individual measurements of parameters $u$ and $q$ [23]. The larger of these two sets of errors was adopted as the random errors $\sigma_{u_{r}}$ and $\sigma_{q_{r}}$ for each night. The final errors $\sigma_{u}$ and $\sigma_{q}$ were calculated from the expressions

$$
\begin{aligned}
\sigma_{u}^{2} & =\sigma_{u_{r}}^{2}+\sigma_{u_{\text {inst }}}^{2} \\
\sigma_{q}^{2} & =\sigma_{q_{r}}^{2}+\sigma_{q_{\text {inst }}}^{2}
\end{aligned}
$$

where $\sigma_{u_{\text {inst }}}$ and $\sigma_{q_{\text {inst }}}$ are the standard errors of the mean instrumental polarization parameters $\bar{u}_{\text {inst }}$, $\bar{q}_{\text {inst }}$ and did not exceed $0.03 \%$.

The errors $\sigma_{p}$ in polarization degree and $\sigma_{\theta}$ in the position angle measurements were determined using the relations [23]:

$$
\begin{aligned}
\sigma_{p} & =\sqrt{\frac{1}{2}\left(\sigma_{u}^{2}+\sigma_{q}^{2}\right)}, \\
\sigma_{\theta} & =28.65 \frac{\sigma_{p}}{P} .
\end{aligned}
$$

The degree of linear polarization $P_{r}$ with respect to the scattering plane $\varphi$ and the angle $\theta_{r}$ between the polarization plane and the plane perpendicular to the scattering plane $\varphi$ were calculated according to the expressions [32]:

$$
\begin{aligned}
P_{r} & =P \cos 2 \theta_{r}, \\
\theta_{r} & =\theta-\left(\varphi \pm 90^{\circ}\right) .
\end{aligned}
$$

\section{DISCUSSION}

Fig. 1 shows the phase-angle dependence of linear polarization for Enceladus in WR band according to this work (filled circles and diamonds). The solid line is the best fit by a trigonometric polynomial [14]. Also the phase-angle dependence of linear polarization for high-albedo Saturnian satellite Rhea in WR band [31] is shown (open circles and diamonds). Circles represent the data for leading hemispheres, and diamonds for trailing hemispheres. Small squares are the laboratory measurements of the phase-angle dependence of the polarization for $\mathrm{MgO}[15]$. As one 
can see, the phase-angle dependence of linear polarization for Enceladus is fairly asymmetrical with the minimal polarization $P_{\min } \approx-0.51 \%$ at the phase angle $\alpha_{\min } \approx 2.4^{\circ}$. The polarimetric observations of Enceladus were made at the larger phase angles compared to the angle where the brightness opposition effect was observed [18, 25].

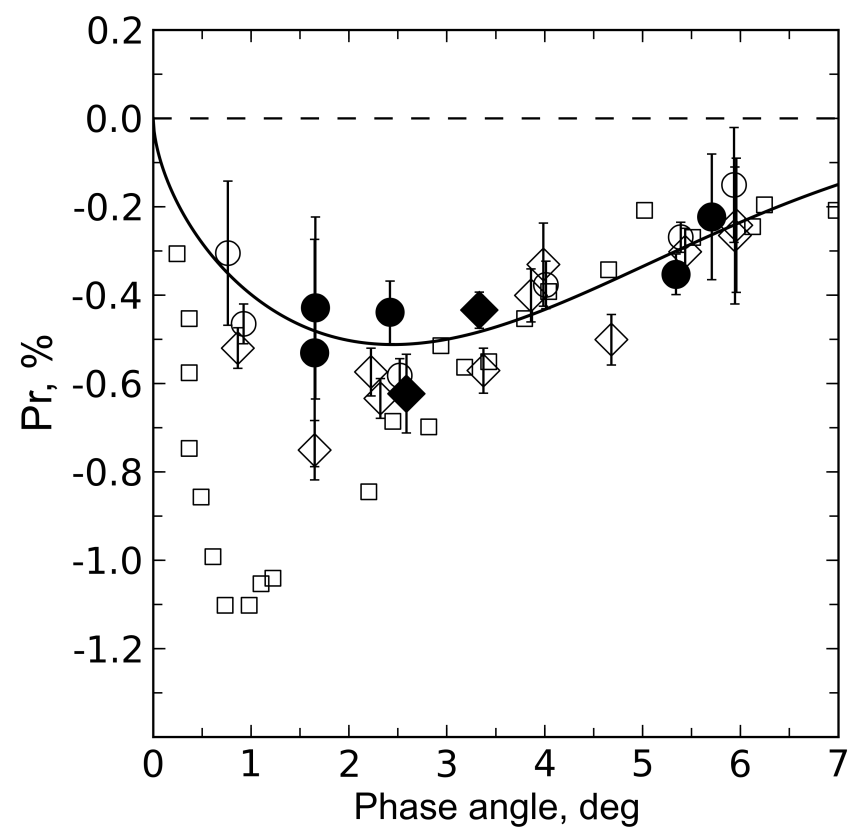

Fig. 1: Phase-angle dependence of polarization for Enceladus (filled circles and diamonds), Rhea (open circles and diamonds), and $\mathrm{MgO}$ powder (small squares).

Fig. 2 shows the longitude dependence of polarization of Enceladus for the interval of phase angles $1^{\circ}-6^{\circ}$ obtained using deviations $\Delta P_{r}$ of the observed polarization from the best fit curve. The differences between the data for leading $\left(L \approx 90^{\circ}\right)$ and trailing $\left(L \approx 270^{\circ}\right)$ hemispheres are within the measurements errors. It can be tentatively concluded that there are no differences in polarimetric properties between the leading and trailing hemispheres.

The form of the phase-angle dependence of linear polarization for Enceladus and its parameters are very close to those obtained for Rhea $\left(P_{\min } \approx-0.6 \%\right.$ and $\alpha_{\min } \approx 2.2^{\circ}$ ). Meanwhile, geometrical albedos of Rhea's leading and trailing side are 0.87 and 0.74 [18], respectively, which are different from that of Enceladus. At the same time, the phase-angle dependence of polarization for Enceladus differs significantly from those measured for $\mathrm{MgO}$ despite their similar albedos.

The phase-angle dependencies of linear polarization for Enceladus and Rhea differs considerably from those for high-albedo Jovian satellites Io, Europa, Ganymede [20, 21, 30], E-type asteroids 44 Nysa [22] and 64 Angelina [22, 29] which demonstrate polarization opposition effect in a form of nar- row secondary minimum of negative polarization at small phase angles. In comparison with the trailing hemisphere of Iapetus [8] having albedo 0.55 in visible light, the icy satellites Rhea and Enceladus show lower asymmetry and less depth of the phase-angle dependence of polarization. Also, Rhea and Enceladus show less depth of the phase-angle dependence of polarization in comparison with large transneptunian objects with icy surfaces $[1,2]$.

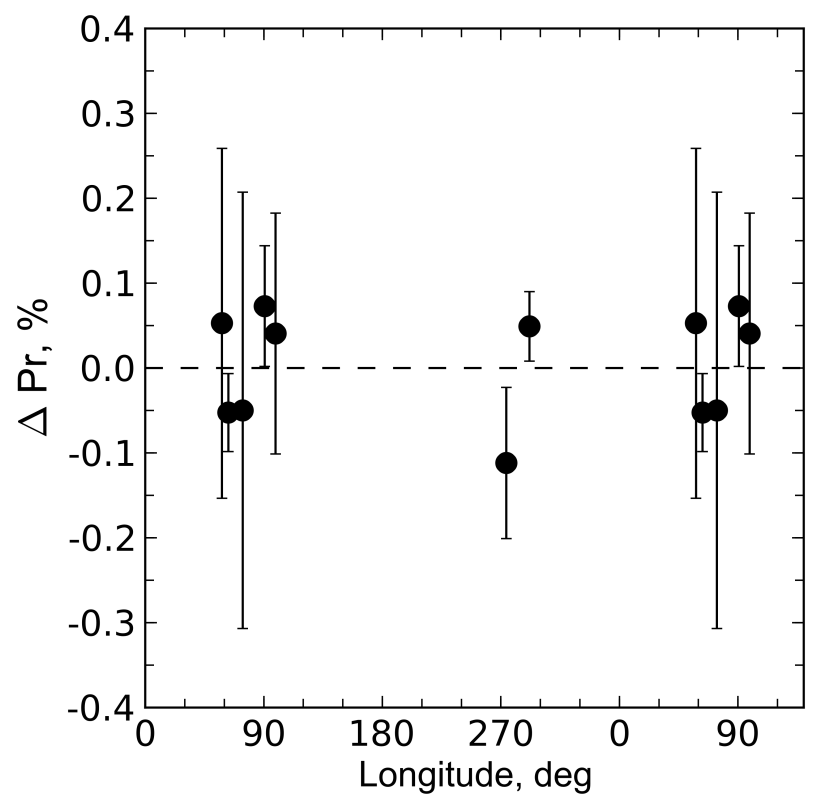

Fig. 2: Longitude dependence of polarization for Enceladus.

Thus, polarimetrical observations of high-albedo Saturnian satellites Enceladus, Rhea, bright side of Iapetus, Jovian satellites Io, Europa, Ganymede, Etype asteroids 44 Nysa and 64 Angelina demonstrate a great diversity in the characteristics of the phaseangle dependence of linear polarization. It can be concluded that these distinctions in the polarization behaviour cannot be explained only by its association with the surface albedo. It is known that the negative polarization branch can be induced by both single scattering by non-spherical grains of the surface regolith layer and near-field effects [17]. The latter mechanism is more effective for low-albedo surfaces with closely packed regolith particles. On the other hand, it was concluded in [6] that both the brightness and polarization opposition effects can be caused by coherent backscattering of solar light even in case of densely packed scattering media like regolith surface layers of high-albedo atmosphereless bodies of the Solar system. To estimate the relative contributions of the mentioned mechanisms to the forming of the phase-angle dependencies of linear polarization and brightness for bodies like Enceladus and Rhea, additional observations in wide range of phase angles and wavelengths are needed. 


\section{CONCLUSIONS}

In this paper, the first polarimetrical observations of Saturnian satellite Enceladus were presented. The phase-angle dependence of linear polarization of the satellite is fairly asymmetrical with the minimal polarization $P_{\min } \approx-0.51 \%$ at the phase angle $\alpha_{\min } \approx$ $2.4^{\circ}$. Its characteristics are very similar to those of the high-albedo Saturnian satellite Rhea. The highalbedo Saturnian satellites Enceladus and Rhea, the bright side of Iapetus, the Jovian satellites Io, Europa, Ganymede, and E-type asteroids 44 Nysa and 64 Angelina demonstrate a great diversity in the characteristics of the phase-angle dependence of linear polarization. It confirms that the polarization behaviour depends not only on albedo but also on the sizes of the reflecting surface's particles and packing density.

\section{REFERENCES}

[1] Bagnulo S., Belskaya I., Boehnhardt H. et al. 2011, J. Quantitative Spectroscopy \& Radiative Transfer, 112, 2059

[2] Belskaya I. N., BagnuloS., Stinson A. et al. 2012, A\&A, 547, A101

[3] Clark R. N., SteeleA., Brown R.H. \& Owensby P. D. 1984, Icarus, 58, 265

[4] Cruikshank D. P. 1980, Icarus, 41, 246

[5] Cruikshank D.P., Owen T. C., Dalle Ore C. et al. 2005, Icarus, 175,268

[6] Dlugach Zh. M. \& Mishchenko M. I. 2013, Solar System Res, 47, 454

[7] Dougherty M. K., Khurana K. K., Neubauer F. M. et al. 2006, Science, 311, 1406

[8] Ejeta C., Boehnhardt H., Bagnulo S. \& Tozzi G. P. 2012, A\&A, 537, A23

[9] Emery J. P., Burr D. M., Cruikshank D. P., Brown R. H. \& Dalton J. B. 2005, ApJ, 435, 353

[10] Geake J. E. \& Geake M. 1990, MNRAS, 245, 46

[11] Heiles C. 2000, AJ, 119, 923

[12] Hendrix A. R., Hansen C. J. \& Holsclaw G. M. 2010, Icarus, 206, 608
[13] Johnson P. E., Kemp J. C., King R., Parker T. E. \& Barbour M. S. 1980, Nature, 283, 146

[14] Lumme K. \& Muinonen K. O. 1993, in 'Abstracts for the IAU Symp. 160: Asteroids, Comets, Meteors 1993', eds.: Harris A. \& Bowell E., Lunar and Planetary Inst., Houston, 194

[15] Lyot B. 1929, Ann. Obs. Paris, 8, 1

[16] Mishchenko M. I. 1993, ApJ, 411, 351

[17] Petrova E. V. \& Tishkovets V. P. 2011, Solar System Res, 45, 304

[18] Pitman K. M., Buratti B. J. \& Mosher J. A. 2010, Icarus, 206, 537

[19] Porco C. C., Helfenstein P., Thomas P. C. et al. 2006, Science, 311, 1393

[20] Rosenbush V.K., Avramchuk V. V., Rosenbush A. E. \& Mishchenko M. I. 1997, ApJ, 487, 402

[21] Rosenbush V., Kiselev N., Avramchuk V. \& Mishchenko M. 2002, in 'Optics of Cosmic Dust, Proc. of a NATO Advanced Research Workshop', Kluwer Academic, Dordrecht, 191

[22] Rosenbush V. K., Shevchenko V. G., Kiselev N. N. et al. 2009, Icarus, 201, 655

[23] Shakhovskoj N. M. \& Efimov Yu. S. 1972, Izvestiya Krymskoj Astrofizicheskoj Observatorii, 45, 90

[24] Spencer J. R., Pearl J. C., Segura M. et al. 2006, Science, 311, 1401

[25] Verbiscer A. J., French R. G. \& McGhee C. A. 2005, Icarus, 173,66

[26] Verbiscer A. J., Peterson D. E., Skrutskie M. F. et al. 2006, Icarus, 182, 211

[27] Waite J.H., Combi M.R., Ip W.-H. et al. 2006, Science, 311,1419

[28] Yeoh S. K., Chapman T. A., Goldstein D. B. et al. 2015, Icarus, 253, 205

[29] Zaitsev S., Kiselev N., Rosenbush V., Kolesnikov S. \& Antonyuk K. 2014, Kinematics and Physics of Celestial Bodies, 30, 155

[30] Zaitsev S. V., Kiselev N. N., Rosenbush V. K. et al. 2012, Advances in Astronomy and Space Physics, 2, 177

[31] Zaitsev S., Kiselev N., Rosenbush V. \& Kolesnikov S. 2015, Kinematics and Physics of Celestial Bodies, 31, 23

[32] Zellner B., Gehrels T. \& Gradie J. 1974, AJ, 79, 1100 